\title{
Unipolar Space-Charge-Limited Current in Solids with Nonuniform Spacial Distribution of Shallow Traps
}

\author{
M-A. Nicolet \\ California Institute of Technology, Pasadena, California
}

(Received 24 January 1966)

\begin{abstract}
Models and analyses of unipolar space-charge-limited current (sclc) in the presence of traps usually assume that the distribution of the traps is uniform in space. It is demonstrated here that this assumption can be a critical one. Simple models with shallow traps are analyzed in detail to establish the fact. Planar, cylindrical, and spherical geometries receive equal attention. The results show that traps in the bulk of the base outside of the immediate vicinity of the emitter are of little significance to the $V-I$ characteristic. The origin of this effect is discussed. A simple approximate treatment is derived. The results imply that the dc characteristic alone is an ambiguous tool for characterizing traps and their physical origin. Included as a byproduct are complete solutions of the $V-I$ characteristics of trap-free sclc in all five distinct cases of the planar, cylindrical, and spherical geometries. Results are given both analytically as well as graphically.
\end{abstract}

\section{INTRODUCTION}

$\mathbf{N}$ UMEROUS experimental investigations have established that unipolar space-charge-limited current (sclc) in solids is normally dominated by traps. The physical identity of these traps remains uncertain. But models have been proposed which describe the experimental facts well in many instances. This success has led to the belief that traps actually do exist in reality with properties much the same as stipulated by the models.

The validity of this conclusion has to be established by detailed experiments and must culminate in the formulation of a microscopic theory of traps and their properties. But this conclusion can also be tested on self-consistency. If a variety of models predict experimentally indistinguishable results the corresponding experiment is obviously not conclusive. It provides, at best, a selection of alternative interpretations. Correspondingly, the nature of the traps remains uncertain within the variations of these models.

Such a situation prevails in experiments on the $V-I$ characteristic of unipolar sclc in solids with shallow traps. These traps are frequently invoked in the literature without further reference to this difficulty. The present study has therefore been undertaken in an effort to shed some light on the subject.

Firstly, a systematic classification of models by the distribution of their traps is developed. This part is general in its character and shows that the models considered so far in the literature constitute but a small fraction of all possible or relevant cases. Secondly, a few selected models possessing shallow traps only are evaluated in detail. Planar, cylindrical, and spherical geometries receive equal attention in this analysis in view of the practical importance of the first and the last one of these cases. The results are also given in graphical form to facilitate discussion and comparison. Included as a by-product are complete solutions of the $V-I$ characteristics of trap-free sclc in all five distinct cases of the three geometries. It is found that the parameters derived for a trap can depend critically on the dis- tribution of the traps assumed in space. Finally, the cause of this effect is discussed and approximate treatments are developed. They yield good results with little effort. Some implications for models with traps other than shallow are briefly considered also.

\section{CLASSIFICATION OF MODELS}

One of the models most frequently encountered in the literature is that of a solid with only one type of trap distributed uniformly in space (density $n_{t}$ ) and discretely in a single energy level (trapping energy $E_{t}$ ). This example illustrates two pertinent facts: (1) To characterize a model at steady state the traps have to be specified in their distribution both in space and in energy. (2) It suggests that among the infinite variety of conceivable cases, the distribution in a unique singularity (on a point, on a line, or on a surface in space and on a point in energy) and the distribution in a continuous and uniform band (over a certain range in space or in energy) are two limiting cases worthy of particular attention. The logical extension of a classification of models according to the distribution of traps eventually leads to a scheme such as that of Table I. The numbers and letters reported in this table give the references to authors having proposed or used a particular model. It is seen that with only two exceptions the distribution of traps has invariably been assumed to be uniform in space, but that a variety of distributions have been considered in energy.

Table I reveals, however, that models with uniform spacial distribution of traps are but one possibility. There are other simple cases such as solids with traps of a single energy level distributed in space on lines, on two-dimensional surfaces, or in selected ranges of volume. There is no reason other than that of convenience why such nonuniform distributions in space should not be considered. Solids with line defects, slip planes, layered structures, or of polycrystalline forms are likely to conform more closely to such or even more complex models than to those assuming uniformity in space. A complete analysis of all relevant cases in detail is a 
TABLE I. Classification of models for unipolar sclc according to the distribution of traps assumed in space and in energy. The letters and numbers give the references to authors having proposed and to some having used a particular model. Papers primarily concerned with subjects other, but related to, unipolar sclc (e.g. photoconductivity, bipolar sclc, contact phenomena) are not included.

\begin{tabular}{|c|c|c|c|c|c|c|c|c|c|c|}
\hline \multirow{2}{*}{\multicolumn{2}{|c|}{$\begin{array}{l}\text { Distribution } \\
\text { in energy } \\
\text { Distribution } \\
\text { in space }\end{array}$}} & \multirow{2}{*}{$\begin{array}{c}\text { in a } \\
\text { point }\end{array}$} & \multirow{2}{*}{$\begin{array}{l}\text { in } \\
\text { points }\end{array}$} & \multicolumn{2}{|c|}{ in a band } & \multicolumn{2}{|c|}{ in bands } & \multirow{2}{*}{$\begin{array}{c}\text { simple or } \\
\text { multiple } \\
\text { combinations } \\
\text { thereof }\end{array}$} & \multicolumn{2}{|c|}{$\begin{array}{l}\text { throughout } \\
\text { all energies }\end{array}$} \\
\hline & & & & uniform & uniform & uniform & uniform & & uniform & uniform \\
\hline \multicolumn{2}{|c|}{$\begin{array}{l}\text { individual } \\
\text { localized point (s) }\end{array}$} & & & & & & & & & \\
\hline $\begin{array}{l}\text { along } \\
\text { line(s) }\end{array}$ & $\begin{array}{l}\text { uniform } \\
\text { non } \\
\text { uniform }\end{array}$ & & & & & & & & & \\
\hline $\begin{array}{l}\text { on } \\
\text { surface (s) }\end{array}$ & $\begin{array}{l}\text { uniform } \\
\text { non- } \\
\text { uniform }\end{array}$ & $\mathbf{a}$ & $r$ & & & & & & & \\
\hline $\begin{array}{l}\text { in } \\
\text { restricted } \\
\text { volume(s) }\end{array}$ & $\begin{array}{l}\text { uniform } \\
\text { non- } \\
\text { uniform }\end{array}$ & & & & & & & & & \\
\hline \multicolumn{2}{|c|}{$\begin{array}{l}\text { simple or multiple } \\
\text { combinations } \\
\text { thereof }\end{array}$} & & & & & & & & & \\
\hline \multirow[t]{2}{*}{$\begin{array}{l}\text { throughout } \\
\text { all space }\end{array}$} & uniform & $1,2, a-q$ & $2, \mathrm{~h}, \mathrm{~s}-\mathrm{x}$ & $\begin{array}{l}1, y, z, b \\
h, j, n\end{array}$ & $\mathrm{~d}, \mathrm{y}$ & $1, u, v$ & & $a a, b, d, l$ & $h, n$ & $\begin{array}{c}1, \mathrm{~h}, \mathrm{j}, \mathrm{l}, \mathrm{n}, \mathrm{w}, \\
\mathrm{ab}, \mathrm{ac}\end{array}$ \\
\hline & $\begin{array}{l}\text { non- } \\
\text { uniform }\end{array}$ & $\mathbf{a}$ & $\mathbf{r}$ & & & & & & & \\
\hline
\end{tabular}

\footnotetext{
i C. Rhys-Roberts and R. H. Tredgold, Proc. Phys. Soc. (London) 76, 497 (1960).

b G. T. Wright, Proc. Inst. Elec. Engrs. (London) B106, 915 (1959).

R. W. Smith, RCA Rev. 20,69 (1959).

d J. J. Brophy and R. J. Robinson, Phys. Rev. 117, 738 (1960).

- G. T. Wright, Solid-State Electr. 2, 165 (1961).

A. Many, S. Z. Weisz, and M. Simhony, Phys. Rev. 126, 1989 (1962).

8 . Many and G. Rakavy. Phys. Rev. 126, 1980 (1962).

h R. S. Muller, C. I. T. Tech. Rept., Div. Engr. Sci. (1962).

i G. H. Heilmeier and P. J. Warter, Phys. Today 15, 30 (1962).

R. S. Muller, Solid-State Electron. 6. 25 (1963).

kM. I. Korsunskii and N. S. Pastushuk, Soviet Phys,-Solid State 5, 407 (1963).

1J. Adolph, E. Baldinger, W. Czaja, and I. Graenacher, Phys. Letters 6 ,

137 (1963). Roberts and R. H. Tredgold, J. Phys. Chem. Solids 25, 1349

(1964). Helfrich, Phys. Status Solidi 7, 863 (1964).
}

huge, tedious and difficult task. The considerations to follow apply to a small selection of specific cases. In addition, the treatment is limited to the simple limit of shallow traps in an insulator.

\section{SHALLOW TRAPS IN PERFECT INSULATOR}

As suggested first by Rose, ${ }^{1}$ it is convenient to classify traps also by the position of their energy level with respect to the steady-state quasi-Fermi level $E_{F}$ of the free charge carriers. Deep traps are located below $E_{F}$ by at least several $k T$; medial traps have energy levels within at least several $k T$ of $E_{F}$; shallow traps are located above $E_{k}$ by at least several $k T$. This distinction is convenient because models can be analysed more readily in both the limiting cases of deep and shallow traps than in the intermediate one. There are, correspondingly, two limiting versions of Table $I$ in which all traps listed are either exclusively deep or exclusively shallow. Only the latter case is retained in the analysis to follow.

\footnotetext{
${ }^{1}$ A. Rose, RCA Rev. 12, 362 (1952).
}

- B. L. Gregory and A. G. Jordan, Phys. Rev, 134A 1378 (1964).

D K. V. Shalimova, L. P. Pavlov, and R. R. Rezvyi, Soviet Phys.-Solid State 6,1749 (1965).

q J. M. Brown and A. G. Jordan, J. Appl. Phys. 36, 337 (1966) . J. Brophy, Phys. Rev. 119, 591 (1960).

- J. A. Hornbeck and J. R. Haynes, Phys. Rev, 97, 311 (1955)

H. P. D. Layon and W. E. Speat, Proc. Phys. Soc. (London) 77, 1157 (1961).

u J. L. Hartke, Phys. Rev. 125, 1177 (1962)

$\checkmark$ A. A. Kastal'skii, Soviet Phys.-Solid State 5, 528 (1963)

M. A. Lampert, A. Many, and P. Mark, Phys. Rev. 135A, 1444 (1964).

x M. A. Lampert, J. Appl. Phys. 35, 2971 (1964).

y A. Rose, Phys. Rev, 97, 1538 (1955)

- R. W. Smith and A. Rose, Phys. Rev. 97, 1531 (1955)

an W. Ruppel, Helv. Phys. Acta 31, 311 (1958).

ab P. Mark and W. Helfrich, J. Appl. Phys. 33, 205 (1962).

Shallow traps merely reduce sclc by a constant factor $F<1$, as was shown by Rose $^{1}$ and Lampert. ${ }^{2,3}$ The "trapping factor" $F$ introduced here depends upon the effective density of states of the conducting band and the distribution of the traps in space and energy; thus

$$
I=I_{\infty} F,
$$

where $I_{\infty}$ is the value of sclc in the absence of traps. To compute $I_{\infty}$, the model of a perfect insulator diode proposed first by Mott and Gurney is used here. The major idealizations of this model are: absence of free charge carriers at thermal equilibrium; absence of imperfections of any kind in the solid; absence of diffusion. Under those conditions, sclc is described by the following set of equations at steady state:

$$
\begin{aligned}
-\nabla \varphi & =\mathbf{E} \\
\epsilon \epsilon_{0} \nabla \cdot \mathbf{E} & =\rho
\end{aligned}
$$

${ }^{2}$ M. A. Lampert, Phys. Rev. 103, 1648 (1956).

${ }^{3}$ M. A. Lampert, Rept. Progr. Phys. 27, 329 (1964).

N. F. Mott and R. W. Gurney, Electronic Processes in Ionic Crystals (Oxford University Press, Oxford, England, 1957), 2nd ed., p. 172. 
TABLE II. $E_{b}=$ edge of the conducting band; $N_{b}=$ effective density of states of the conducting band; $n_{t}=\int N(E) d E$ from $E_{t l}$ to $E_{t u}=$ density of trapping states; $\Gamma(n+1, y)=\int_{0}^{y} x^{n} e^{-x} d x ; G(n+1, y)=\int_{0}^{y} x^{n} e^{x} d x ; 2 n ! !=2.4 .6 \cdots(2 n)$; $(2 n-3) ! ! !=1.3 .5 \cdots(2 n-3)$ and $=1$ for $n=1$.

\begin{tabular}{|c|c|}
\hline ENERGY DISTRIBUTION OF SHALLOW TRAPS & TRAPPING PARAMETER \\
\hline $\begin{array}{l}E_{b} \\
\text { by superposition of } k \text { distributions of } \\
\text { known } \theta_{n}(n=1, \cdots, k)\end{array}$ & $\begin{array}{l}\theta^{-1}=\frac{e^{E_{b} / k T}}{N_{b}} \int_{E_{+1}}^{E_{f u}} N(E) e^{-E / k T} d E \\
\theta^{-1}=\sum_{n=1}^{k} \theta_{n}^{-1}\end{array}$ \\
\hline $\begin{array}{l}E_{b} \\
E_{\dagger} \rightleftharpoons n_{\dagger}\end{array}$ & $\theta=\frac{N_{b}}{n_{t}} e^{-\left(E_{b}-E_{1}\right) / k T}$ \\
\hline with $\theta^{-1}=A e^{\left(E_{b}-E_{t 1}\right) / k T}-B e^{\left(E_{b}-\right.}$ & $/ k T$ the factors $A, B$ are: \\
\hline $\begin{array}{l}\text { if } d^{n} N(E) / d E^{n} \equiv N^{(n)}(E) \text { and if the series } \\
\text { converge }\end{array}$ & $\begin{array}{l}A=\left(k T / N_{b}\right) \sum_{n=0}^{\infty}(k T)^{n} N^{(n)}\left(E_{t)}\right) \\
B=\left(k T / N_{b}\right) \sum_{n=0}^{\infty}(k T)^{n} N^{(n)}\left(E_{q u}\right)\end{array}$ \\
\hline \begin{tabular}{l|l}
$E_{b}$ & $N_{0} N_{1}$ \\
$E_{t u}$ &
\end{tabular}$N_{0}+\frac{E-E_{t 1}}{\Delta E} N_{1}$ & $\begin{array}{l}A=\left(k T / N_{b}\right)\left(N_{0}+N_{1} k T / \Delta E\right) \\
B=\left(k T / N_{b}\right)\left(N_{0}+N_{1}+N_{1} k T / \Delta E\right)\end{array}$ \\
\hline$E_{t u}^{E_{b}, f N_{0} N_{1}} N_{0} e^{\left(E-E_{11}\right) / k T_{t}}$ & $\begin{array}{ll}A=\left(k T^{*} / N_{b}\right) N_{0} & T^{*}=\frac{T_{f} T}{T_{f} T} \\
B=\left(k T^{*} / N_{b}\right)\left(N_{0}+N_{1}\right) & \end{array}$ \\
\hline$\underbrace{E_{b}}_{N_{0}} N_{0}\left(\frac{E_{t u}-E}{\Delta E}\right)^{n}$ porabolical $N_{0}\left(\frac{E-E_{t 1}}{\Delta E}\right)^{n}$ & $\begin{array}{l}A=0 \\
B=-\left(N_{0} k T / N_{b}\right)(k T / \Delta E)^{n} G(n+1, \Delta E / k T) \\
A=\left(N_{0} k T / N_{b}\right)(k T / \Delta E)^{n} \Gamma(n+1, \Delta E / k T) \\
B=0\end{array}$ \\
\hline $\begin{array}{l}E_{b} \\
E_{t u} \overbrace{N_{0}} \text { ellipsoidal } \\
E_{01} \sqrt{1-\left(\frac{E-E_{t 1}}{\Delta E}\right)^{2}} \\
E_{b} N_{E_{0}}+N_{0} \sqrt{1-\left(\frac{E_{t u}-E}{\Delta E}\right)^{2}}\end{array}$ & $\begin{array}{l}A=\left(N_{0} k T / N_{b}\right)\left[1-\sum_{n=1}^{\infty} \frac{(2 n-3) ! ! !}{2 n ! !}(k T / \Delta E)^{2 n} \Gamma(2 n+1, \Delta E / k T)\right] \\
B=N_{0} k T / N_{b} \\
A=N_{0} k T / N_{b} \\
B=\left(N_{0} k T / N_{b}\right)\left[1+\sum_{n=1}^{\infty} \frac{(2 n-3) ! ! !}{2 n ! !}(k T / \Delta E)^{2 n} G(2 n+1, \Delta E / k T)\right]\end{array}$ \\
\hline
\end{tabular}

$$
\begin{aligned}
\mathbf{J} & =\rho \mathbf{v} \\
\mathbf{v} & =\mu \mathbf{E} \\
\nabla \cdot J & =0 .
\end{aligned}
$$

With the boundary condition $E$ (emitter) $=0$ the solu-
(4) tion is always of the form ${ }^{5}$

$$
I_{\infty}=\epsilon \epsilon_{0} \mu V^{2} \delta,
$$

(6) where $\mu$ is the charge carrier mobility, $\epsilon$ is the dielectric ${ }^{5}$ D. H. Lee and M-A. Nicolet, Solid-State Electron. 8, 182 (1965). 
constant of the solid, $V$ is the applied voltage, and $\delta$ is a factor which depends only on the geometry of the device. When shallow traps are present in such an otherwise perfect insulator sclc thus always follows the relationship

$$
I=\epsilon \epsilon_{0} \mu V^{2} \delta F \text {. }
$$

The problem of finding the solutions of Table I now reduces to an evaluation of $\delta F$ for a given device geometry and distribution of traps in space and in energy.

As long as the traps are shallow, the ratio $\theta$ of the mobile charge carrier concentration $\rho_{m}$ to the trapped charge carrier concentration $\rho_{t}$ is constant at all operating points. ${ }^{1,2}$ The details of the trap distribution in energy then enters into $F$ only via $\theta$ and the effects of the trap distribution in space and in energy can be discussed separately. Ambiguities in interpretation of an experimental result in terms of particular traps are thus especially pronounced in this case. Table II gives the value of $\theta$ for a selection of energy distributions, assuming equal statistical weight for all levels.

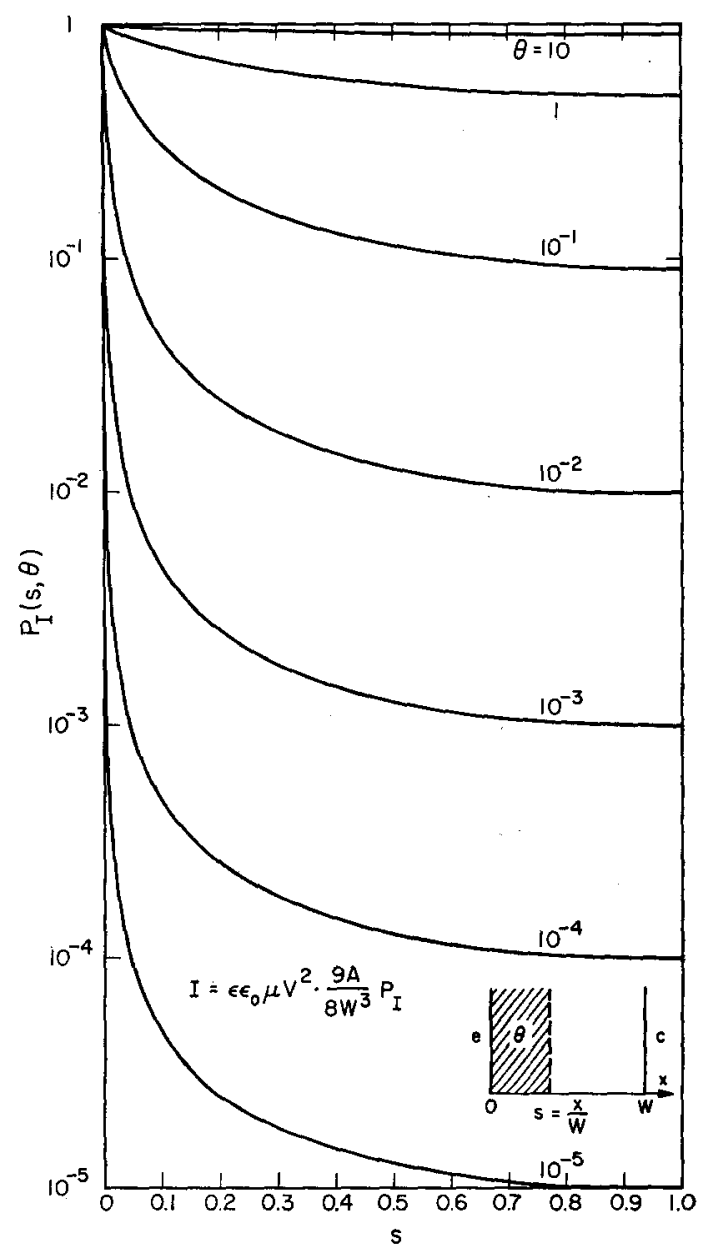

Fig. 1. Shallow traps of trapping parameter $\theta$ distributed uniformly over a limited distance in front of the emitter reduce sclc by a factor $P_{\mathrm{I}}(s, \theta)$ in structures of planar geometry.

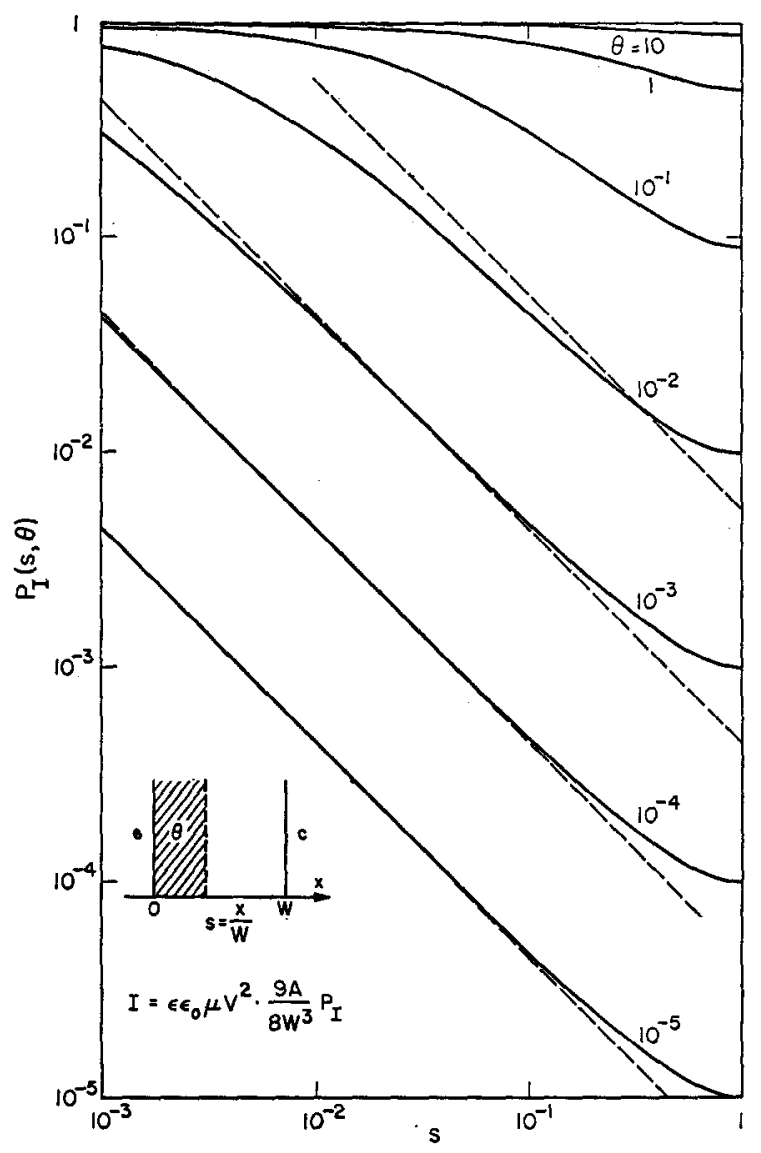

FIG. 2. The dependence of $P_{\mathrm{I}}(s, \theta)$ of Fig. 1 expanded at small values of the trapping layer. Dashed lines are for the approximate treatment.

The following sections evaluate $\delta F$ for a few particular distributions of traps in space, assuming either planar, cylindrical or spherical geometry for the structure of the diode. Solutions are obtained by integrating Eqs. (2)-(6) with the substitutions $\rho=\rho_{m}$ in Eq. (4) and writing the result in the form of Eq. (7).

\section{MODELS OF PLANAR GEOMETRY}

For planar geometry

$$
\delta=\delta^{p} \equiv 9 A / 8 W^{3},
$$

where $A$ is the area of the cross section carrying the current.

\subsection{Uniform Distribution of Shallow Traps over a Limited Distance in Front of the Emitter (Figs. 1 and 2)}

It is assumed that shallow traps of a trapping parameter $\theta$ are distributed uniformly in space and over a distance $0 \leq \phi \leq W$ in front of the emitter. A piecewise integration, matching the electric field at the interface 


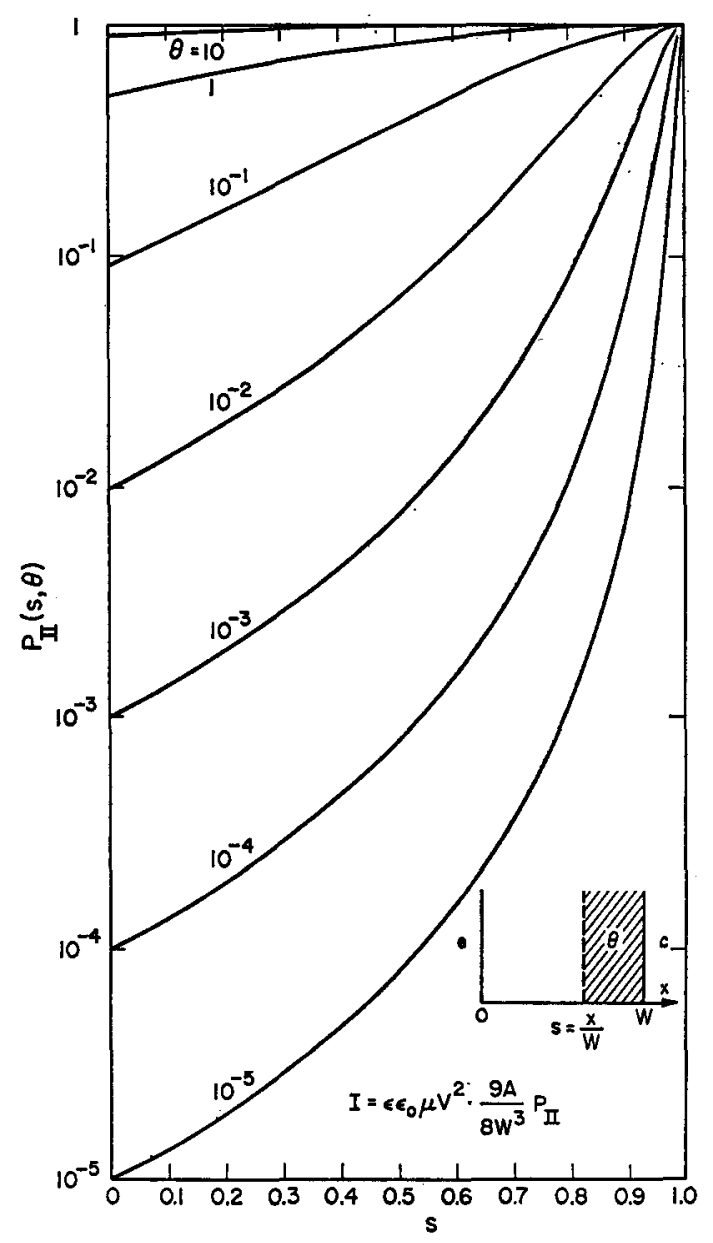

FIG. 3. Shallow traps of trapping parameter $\theta$ distributed uniformly over a limited distance in front of the collector reduce sclc by a factor $P_{\mathrm{II}}(s, \theta)$ in structures of planar geometry.

between regions with and without traps, yields

$$
F=P_{\mathrm{I}} \equiv\left[s^{\frac{3}{3}}\left(1+\theta^{-1}\right)^{\frac{1}{2}}+\left(1+s \theta^{-1}\right)^{\frac{1}{2}}-s^{\frac{3}{2}}\left(1+\theta^{-1}\right)^{\frac{1}{2}}\right]^{-2} .
$$

Values of $P_{\mathrm{I}}(s, \theta)$ are plotted for various $\theta$ in Fig. 1 and 2.

\subsection{Uniform Distribution of Shallow Traps over a Limited Distance in Front of the Collector} (Fig. 3)

In this case, the solution yields

$$
\begin{aligned}
F=P_{\mathrm{II}} \equiv\left[s^{\frac{3}{2}}+\left(1+\theta^{-1}\right)^{-1}\left(1+\theta^{-1}-s \theta^{-1}\right)^{\frac{1}{4}}\right. & \left.-\left(1+\theta^{-1}\right)^{-1} s^{\frac{2}{2}}\right]^{-2} .
\end{aligned}
$$

Values of $P_{\mathrm{II}}(s, \theta)$ are plotted for various $\theta$ in Fig. 3.

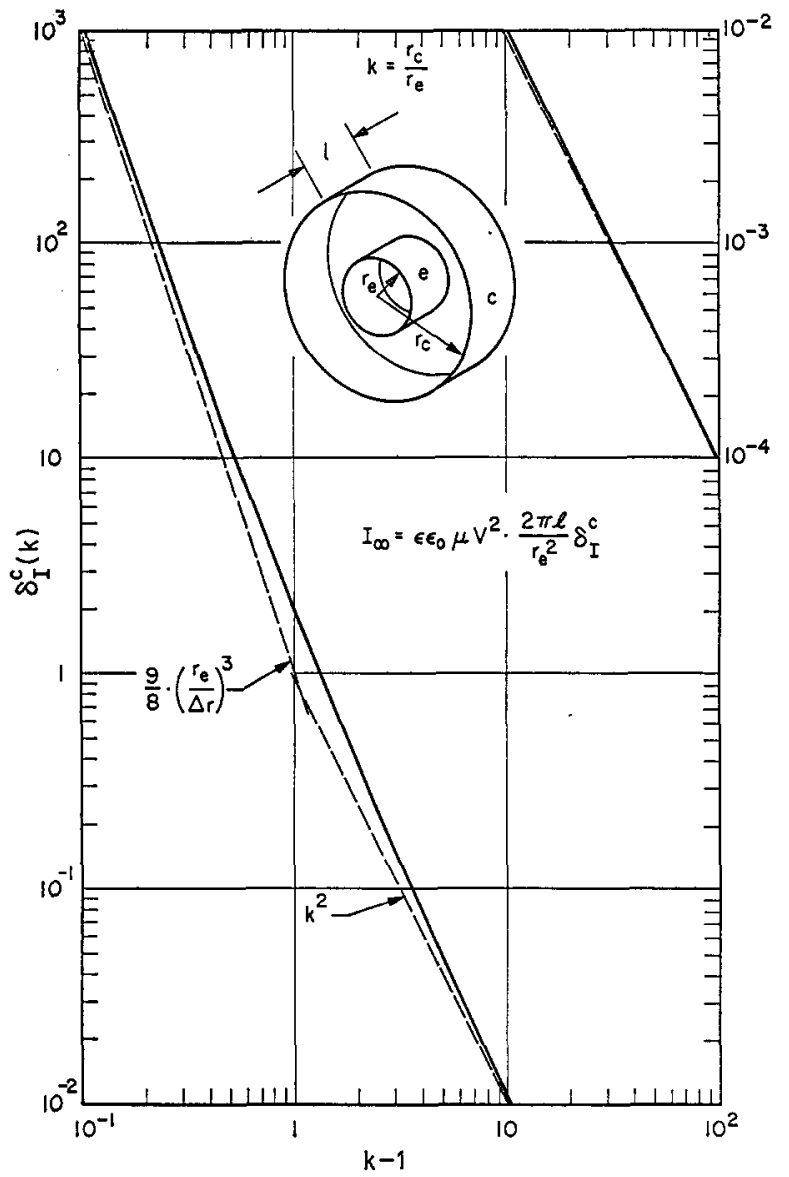

FIG. 4. The geometrical factor $\delta_{\mathrm{I}}^{c}(k)=\delta^{c, i} / 2 \pi l r_{e}^{-2}$ for pure sclc flow through a cylindrical structure with the emitter at the inner electrode. Dashed lines give asymptotic dependences.

\section{MODELS OF CYLINDRICAL GEOMETRY}

Two cases must be distinguished according to the location of the emitter at either the inside or the outside electrode of the cylinder. One finds

for the emitter inside

$$
\delta=\delta^{c, i} \equiv 2 \pi l r_{e}^{-2}\left[\left(k^{2}-1\right)^{\frac{1}{2}}-\operatorname{arcos}\left(k^{-1}\right)\right]^{-2}, \quad(1 \leq k)
$$

for the emitter outside

$$
\delta=\delta^{c, o}=2 \pi \operatorname{lr}_{\mathrm{e}}^{-2}\left[\operatorname{arcosh}\left(k^{-1}\right)-\left(1-k^{2}\right)^{\frac{1}{2}}\right]^{-2}, \quad(k \leq 1)
$$

where $l$ is the length of the cylinder, $r_{e}$ is the emitter radius, and $k r_{e}$ is the collector radius. The dependences of $\delta^{c, i}$ and $\delta^{c, o}$ on $k$ are shown graphically in Figs. 4 and 5 , respectively. Approximations valid for $k \gg 1, k \approx 1$, and $k \ll 1$ are also plotted (dashed lines).

\subsection{Uniform Distribution of Shallow Traps over a Limited Distance in Front of the Emitter}

(Figs. 6 and 7)

The solution with the emitter inside is

$$
F=C_{\mathrm{I}} \equiv \frac{\left[\left(k^{2}-1\right)^{\frac{1}{2}}-\operatorname{arcos}\left(k^{-1}\right)\right]^{2}}{\left\{\left(k^{2}-z^{2}\right)^{\frac{1}{2}}-\left(1+\theta^{-1}\right)^{\frac{1}{3}} \operatorname{arcos}\left(s^{-1}\right)-z[\operatorname{arcos}(z / k)-\operatorname{arcos}(z / s)]\right\}^{2}},
$$


with $z^{2}=\left(1+\theta-s^{2}\right) / \theta$ for $s^{2} \leq 1+\theta$, and

$$
F=C_{\mathrm{I}} \equiv \frac{\left[\left(k^{2}-1\right)^{\frac{1}{3}}-\operatorname{arcos}\left(k^{-1}\right)\right]^{2}}{\left\{\left(k^{2}+y^{2}\right)^{\frac{1}{2}}-\left(1+\theta^{-1}\right)^{\frac{1}{2}} \operatorname{arcos}\left(s^{-1}\right)+y[\operatorname{arsinh}(y / s)-\operatorname{arsinh}(y / k)]\right\}^{2}},
$$

where $y^{2}=\left(s^{2}-1-\theta\right) / \theta$ for $s^{2} \geq 1+\theta$. Values of $C_{\mathrm{I}}(s, \theta, k)$ are plotted in Fig. 6 for $k=10$ and various $\theta$.

The solution with the emitter outside is

$$
F=C_{\mathrm{III}} \equiv \frac{\left[\operatorname{arcosh}\left(k^{-1}\right)-\left(1-k^{2}\right)^{1}\right]^{2}}{\left\{z[\operatorname{arcosh}(z / k)-\operatorname{arcosh}(z / s)]+\left(1+\theta^{-1}\right) \operatorname{arcosh}\left(s^{-1}\right)-\left(z^{2}-k^{2}\right)^{\left.\frac{1}{2}\right\}^{2}}\right.},
$$

where $z^{2}=\left(1+\theta-s^{2}\right) / \theta$. Values of $C_{\mathrm{III}}(s, \theta, k)$ are plotted in Fig. 7 for $k=10^{-1}$ and various $\theta$.

\subsection{Uniform Distribution of Shallow Traps over a Limited Distance in Front of the Collector (Figs. 8 and 9)}

The solution with the emitter inside is

$$
F=C_{\mathrm{II}} \equiv \frac{\left[\left(k^{2}-1\right)^{\frac{1}{2}}-\operatorname{arcos}\left(k^{-1}\right)\right]^{2}}{\left(1+\theta^{-1}\right)\left\{\left(k^{2}-x^{2}\right)^{\frac{1}{3}}-\left(1+\theta^{-1}\right)^{-\frac{1}{3}} \operatorname{arcos}\left(s^{-1}\right)-x[\operatorname{arcos}(x / k)-\operatorname{arcos}(x / s)]\right\}^{2}},
$$

where $x^{2}=\left(s^{2}+\theta\right) /(1+\theta)$. Values of $C_{\mathrm{II}}(s, \theta, k)$ are plotted in Fig. 8 for $k=10$ and various $\theta$.

The solution with the emitter outside is

$$
F=C_{\mathrm{IV}} \equiv \frac{\left[\operatorname{arcosh}\left(k^{-1}\right)-\left(1-k^{2}\right)^{\frac{1}{2}}\right]^{2}}{\left(1+\theta^{-1}\right)\left\{x[\operatorname{arcosh}(x / k)-\operatorname{arcosh}(x / s)]+\left(1+\theta^{-1}\right)^{-\frac{1}{2}} \operatorname{arcosh}\left(s^{-1}\right)-\left(x^{2}-k^{2}\right)^{\frac{1}{1}}\right\}^{2}},
$$

where $x^{2}=\left(s^{2}+\theta\right) /(1+\theta)$. Values of $C_{\mathrm{IV}}(s, \theta, k)$ are plotted in Fig. 9 for $k=10^{-1}$ and various $\theta$.

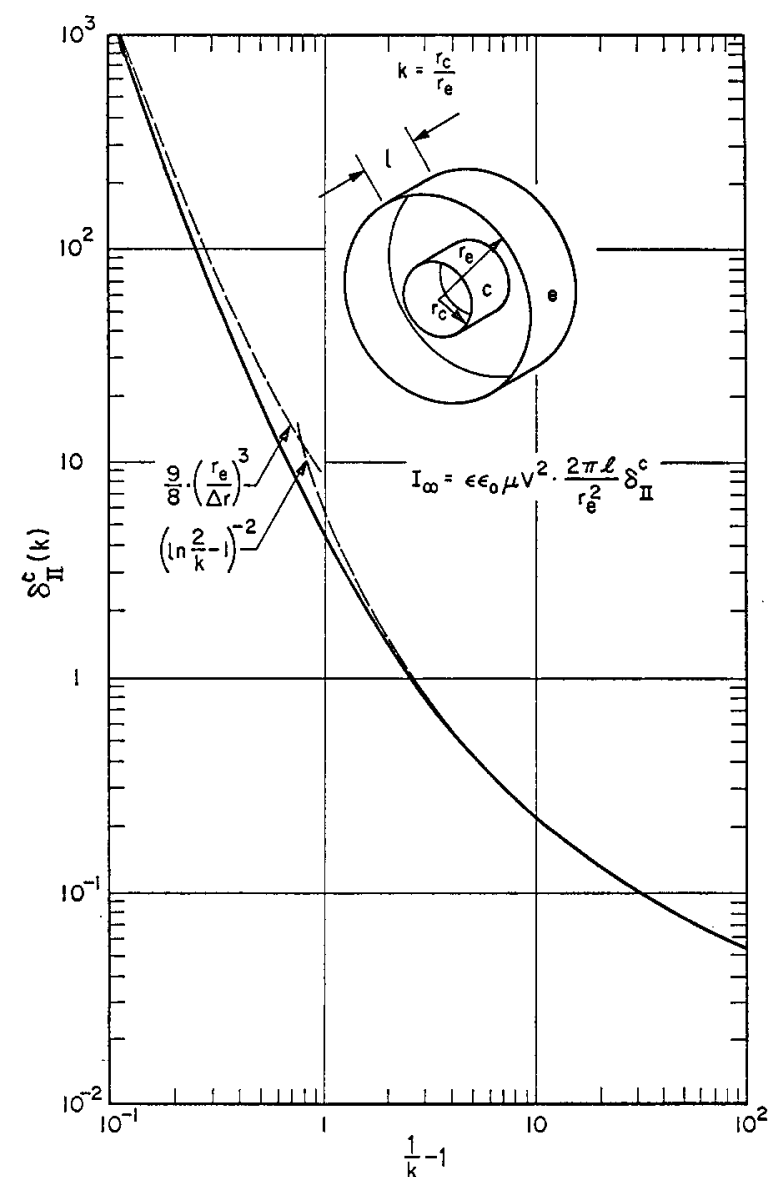

FIG. 5. The geometrical factor $\delta_{\mathrm{II}}^{c}(k)=\delta^{c, 0} / 2 \pi l_{e}{ }^{-2}$ for pure sclc flow through a cylindrical structure with the emitter at the outer electrode. Dashed lines give asymptotic dependences.

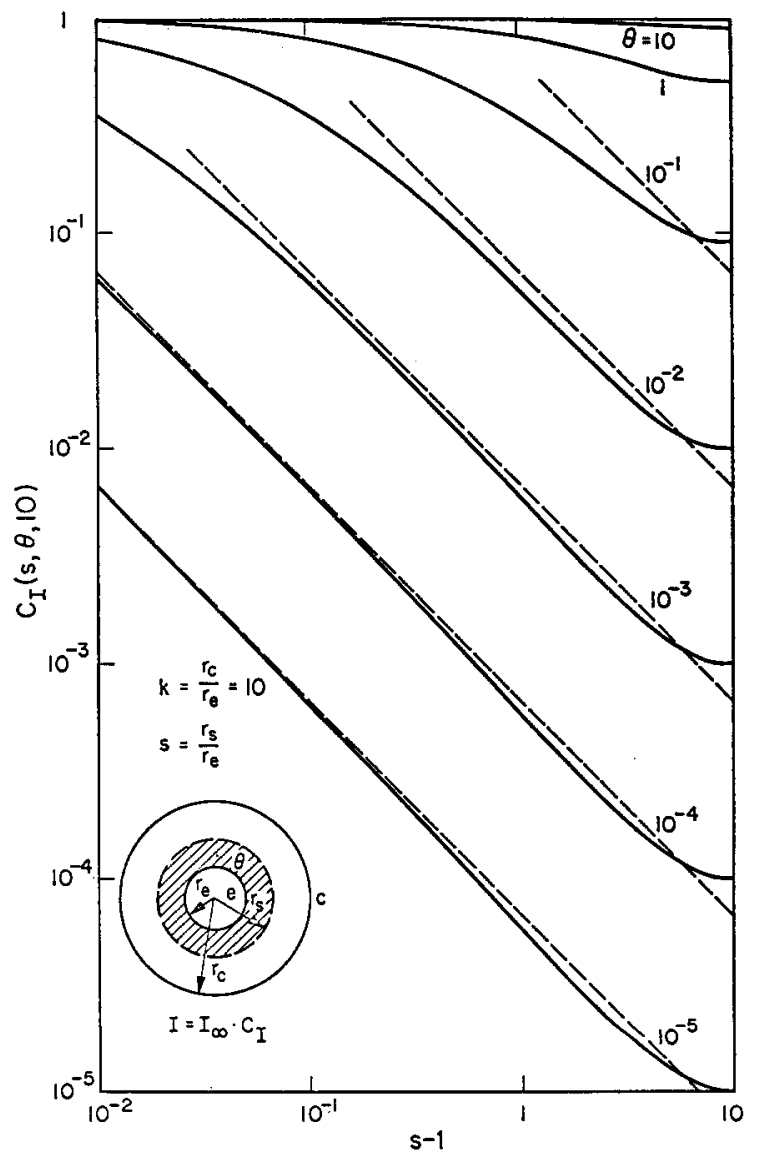

FIG. 6. Shallow traps of trapping parameter $\theta$ distributed uniformly over a limited distance in front of the emitter reduce sclc by a factor $C_{\mathrm{l}}(s, \theta, k)$ in structures of cylindrical geometry with the emitter at the inner electrode. Dashed lines are for the approximate treatment. 

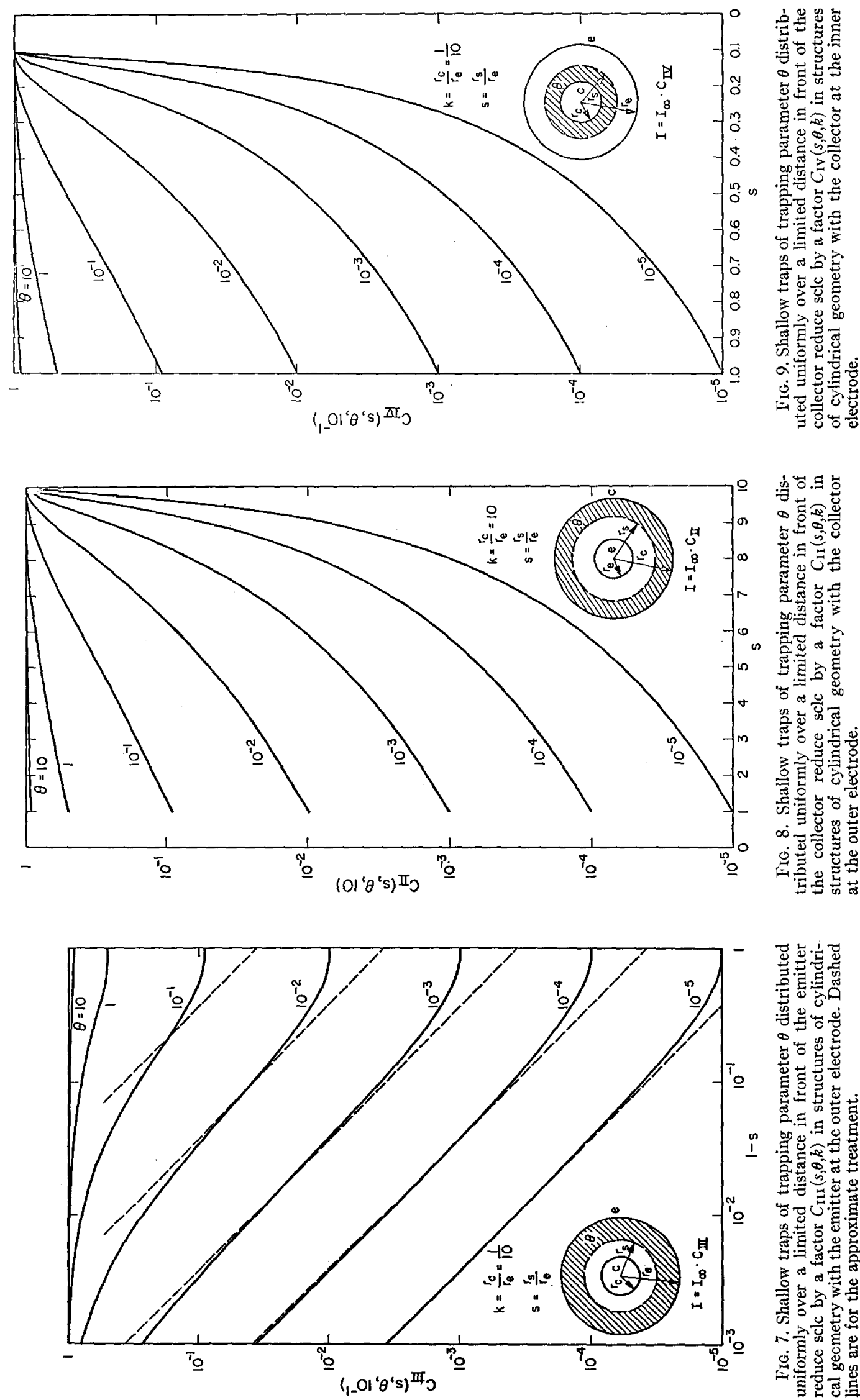


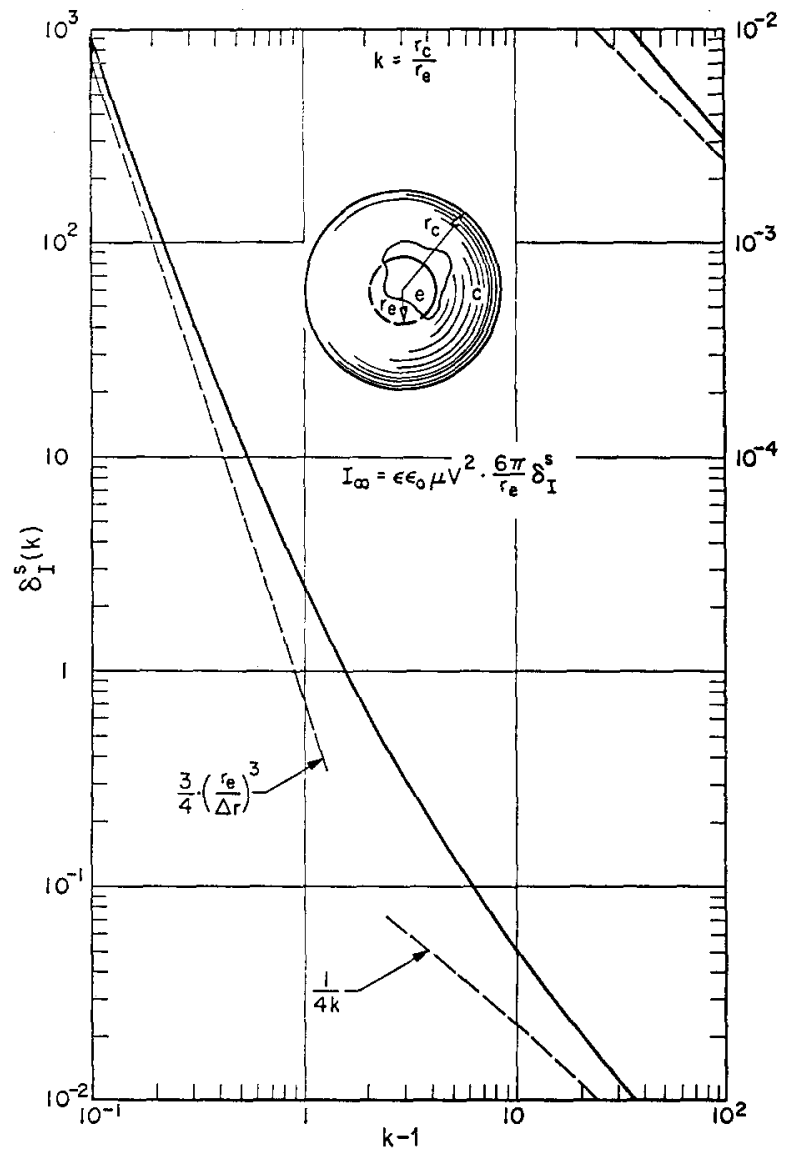

FIG. 10. The geometrical factor $\delta_{1}^{s}(k)=\delta^{s, i} / 6 \pi r_{e}^{-1}$ for pure slc flow through a spherical structure with the emitter at the inner electrode. Dashed lines give asymptotic dependences.

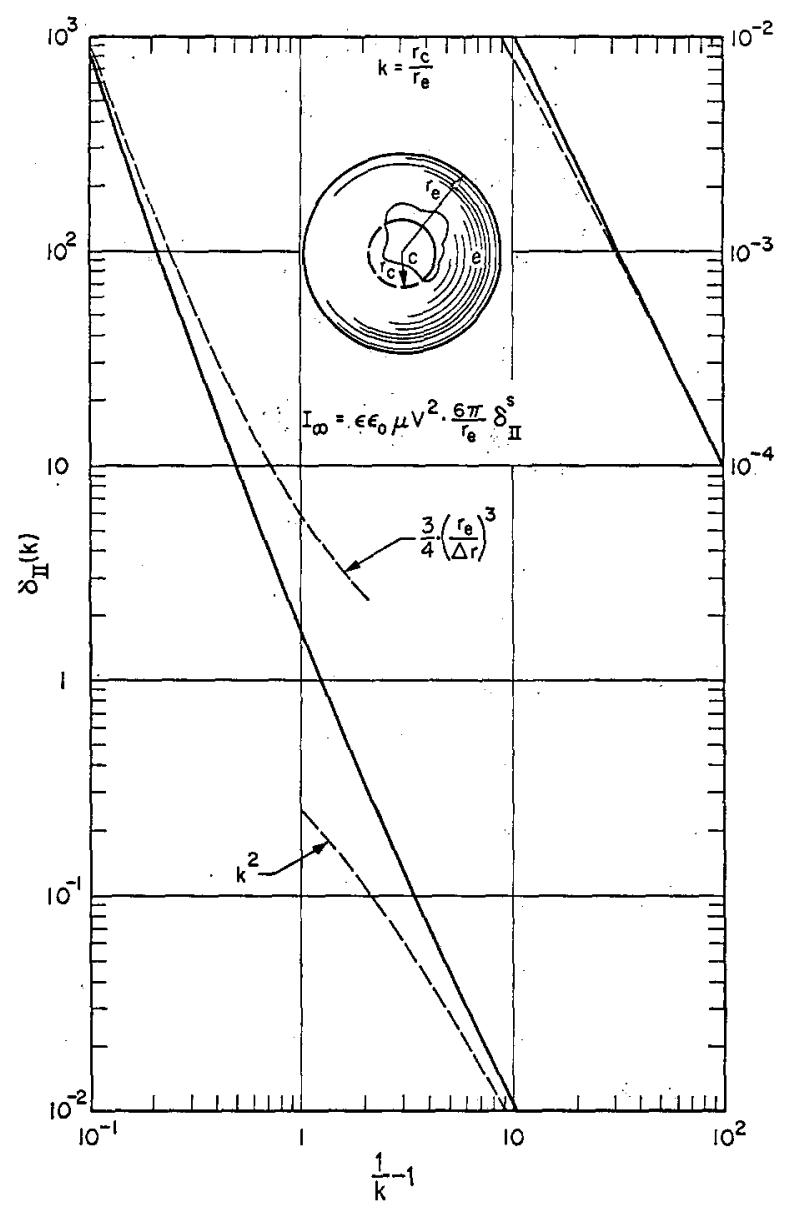

FIG. 11. The geometrical factor $\delta_{\mathrm{II}} I^{8}(k)=\delta^{s, o} / 6 \pi r_{e}^{-1}$ for pure sclc flow through a spherical structure with the emitter at the outer electrode. Dashed lines give asymptotic dependences.

\section{MODELS OF SPHERICAL GEOMETRY}

Two cases must be distinguished according to the location of the emitter at either the inside or the outside electrode of the sphere. One finds

for the emitter inside

$$
\delta=\delta^{s, i} \equiv 6 \pi r_{e}^{-1}\left[\int_{1}^{k}\left(z^{3}-1\right)^{\frac{1}{2}} z^{-2} d z\right]^{-2},(1 \leq k)
$$

for the emitter outside

$$
\delta=\delta^{z, o} \equiv 6 \pi r_{e}^{-1}\left[\int_{k}^{1}\left(1-z^{3}\right)^{\frac{1}{2} z^{-2}} d z\right]^{-2},(k \leq 1)
$$

where $r_{e}$ is the emitter radius and $k r_{e}$ is the collector radius. The dependences of $\delta^{s, i}$ and $\delta^{s, o}$ on $k$ are shown graphically in Figs. 10 and 11, respectively. Approximations valid for $k \gg 1, k \approx 1$ and $k \ll 1$ are also plotted (dashed lines).

\subsection{Uniform Distribution of Shallow Traps over a Limited Distance in Front of the Emitter}

(Figs. 12 and 13)

The solution with the emitter inside is

$$
F=S_{\mathrm{I}}=\frac{\left[\int_{1}^{k}\left(z^{3}-1\right)^{\frac{1}{2}} z^{-2} d z\right]^{2}}{\left\{\left(1+\theta^{-1}\right)^{\frac{1}{2}}\left[\int_{1}^{s}\left(z^{3}-1\right)^{\frac{1}{2}} z^{-2} d z\right]+\int_{s}^{k}\left[\left(1+\theta^{-1}\right)\left(s^{3}-1\right) s^{-4}+\left(z^{3} s^{-3}-1\right) s^{-1}\right]^{\frac{1}{2}}(z / s)^{-2} d z\right\}^{2}} .
$$

Values of $S_{\mathrm{I}}(s, \theta, k)$ are plotted in Fig. 12 for $k=10$ and various $\theta$. 


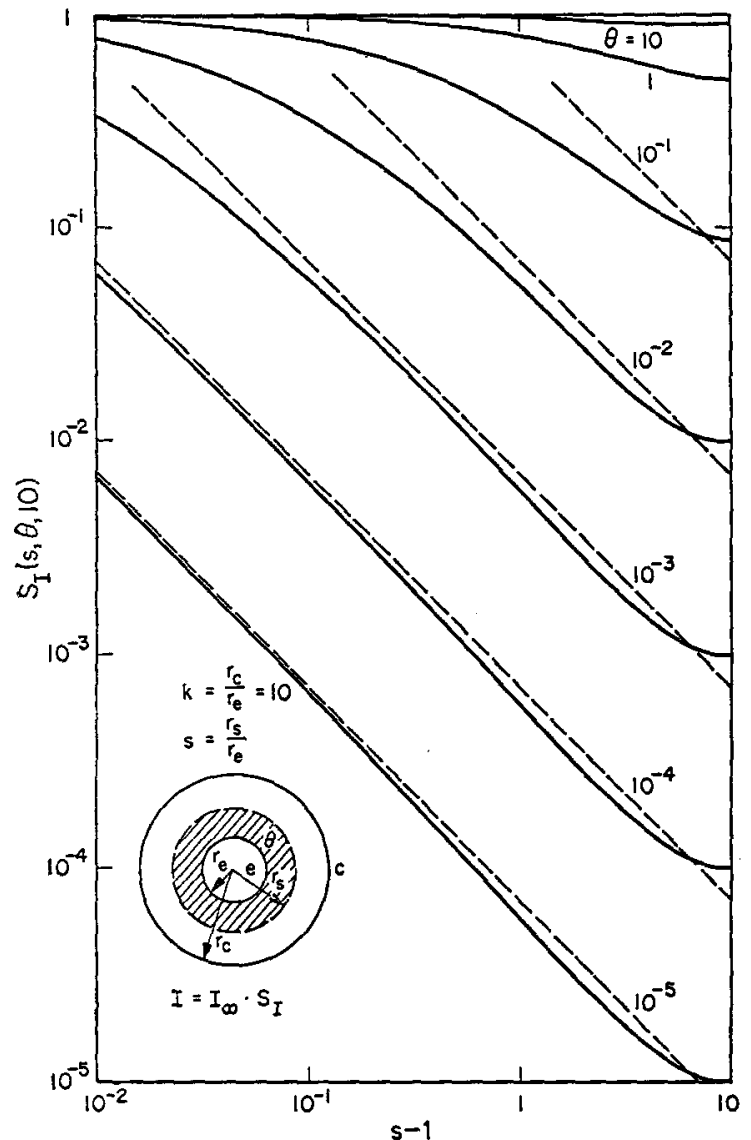

Fig. 12. Shallow traps of trapping parameter $\theta$ distributed uniformly over a limited distance in front of the emitter reduce sclc by a factor $S_{\mathrm{I}}(s, \theta, k)$ in structures of spherical geometry with the emitter at the inner electrode. Dashed lines are for the approximate treatment.

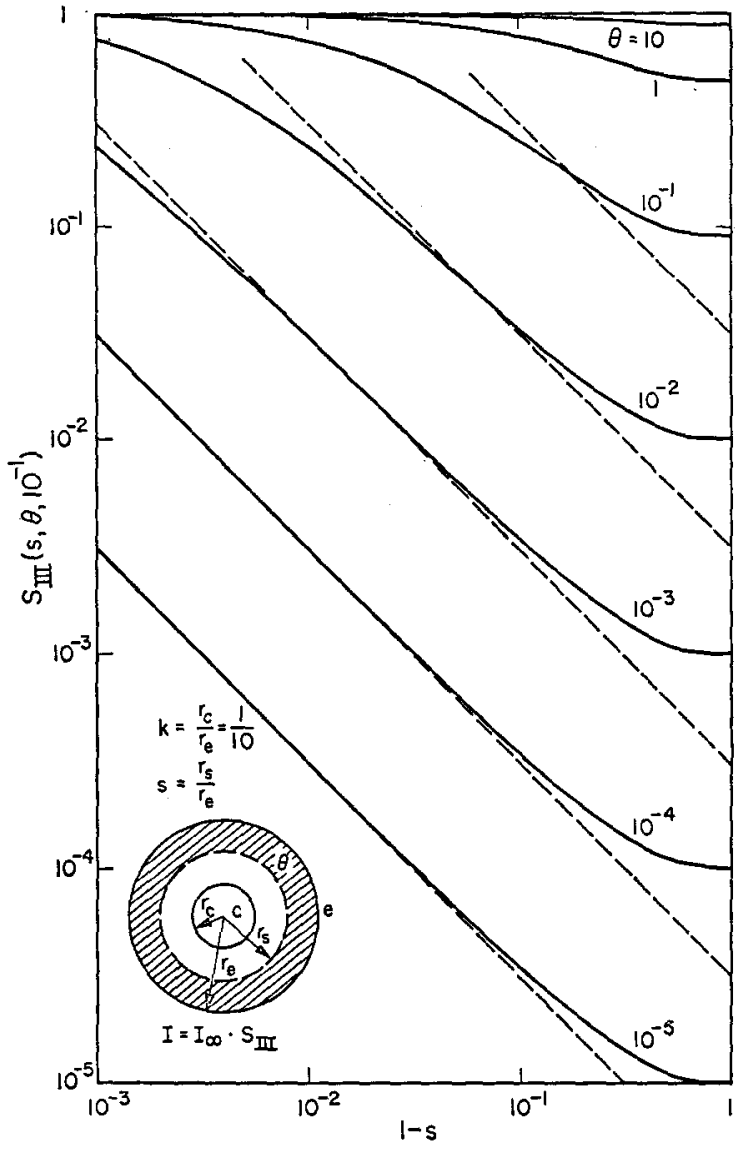

FIG, 13. Shallow traps of trapping parameter $\theta$ distributed uniformly over a limited distance in front of the emitter reduce sclc by a factor $S_{\mathrm{III}}(s, \theta, k)$ in structures of spherical geometry with the emitter at the outer electrode. Dashed lines are for the approximate treatment.

The solution with the emitter outside is

$$
F=S_{\mathrm{III}} \equiv \frac{\left[\int_{k}^{1}\left(1-z^{3}\right)^{\frac{1}{2}} z^{-2} d z\right]^{2}}{\left\{\left(1+\theta^{-1}\right)\left[\int_{8}^{1}\left(1-z^{3}\right)^{\frac{1}{3}} z^{-2} d z\right]+\int_{k}^{s}\left[\left(1+\theta^{-1}\right)\left(1-s^{3}\right)+s^{3}-z^{3}\right]^{\frac{1}{3}} z^{-2} d z\right\}^{2}} .
$$

Values of $S_{\operatorname{III}}(s, \theta, k)$ are plotted in Fig. 13 for $k=10^{-1}$ and various $\theta$.

\subsection{Uniform Distribution of Shallow Traps over a Limited Distance in Front of the Collector}

(Figs. 14 and 15)

The solution with the emitter inside is

$$
F=S_{\mathrm{II}} \equiv \frac{\left[\int_{1}^{k}\left(z^{3}-1\right)^{\frac{1}{z}-2} d z\right]^{2}}{\left\{\int_{1}^{s}\left(z^{3}-1\right)^{\frac{1}{t}} z^{-2} d z+\int_{\delta}^{k}\left[s^{3}-1+\left(1+\theta^{-1}\right)\left(z^{3}-s^{3}\right)\right]^{1} z^{-2} d z\right\}^{2}} .
$$

Values of $S_{\mathrm{II}}(s, \theta, k)$ are plotted in Fig. 14 for $k=10$ and various $\theta$. 


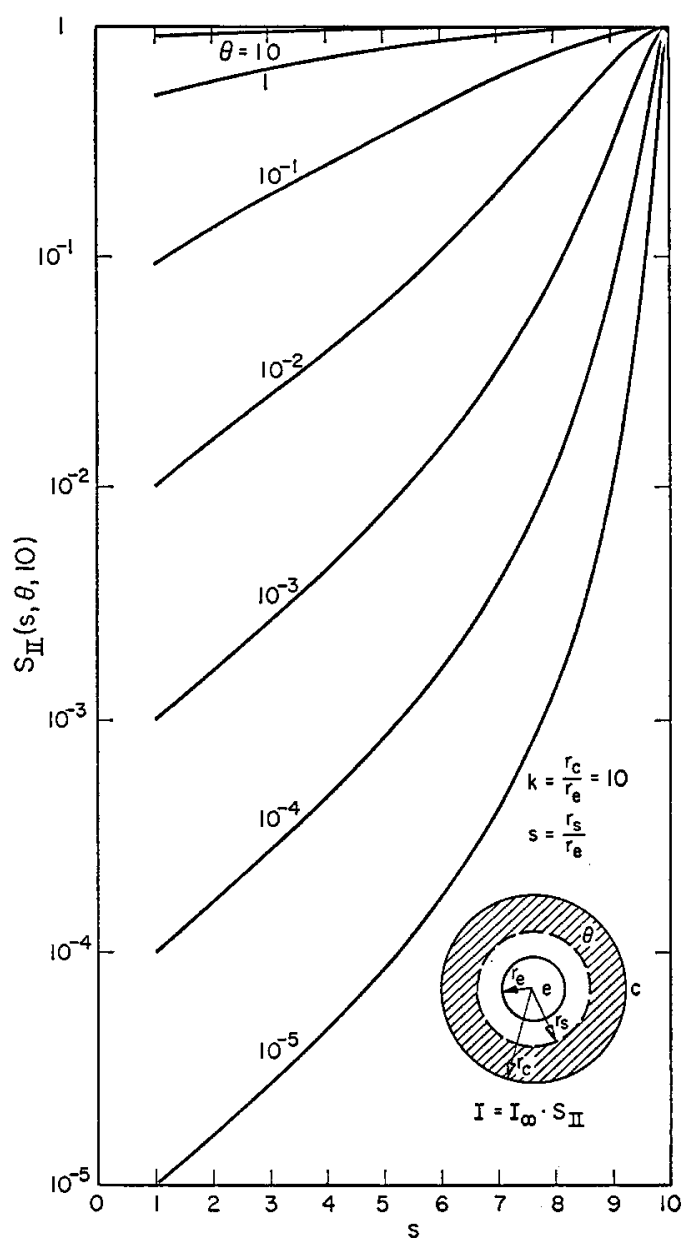

FIG. 14. Shallow traps of trapping parameter $\theta$ distributed uniformly over a limited distance in front of the collector reduce sclc by a factor $S_{\mathrm{II}}(s, \theta, k)$ in structures of spherical geometry with the collector at the outer electrode.

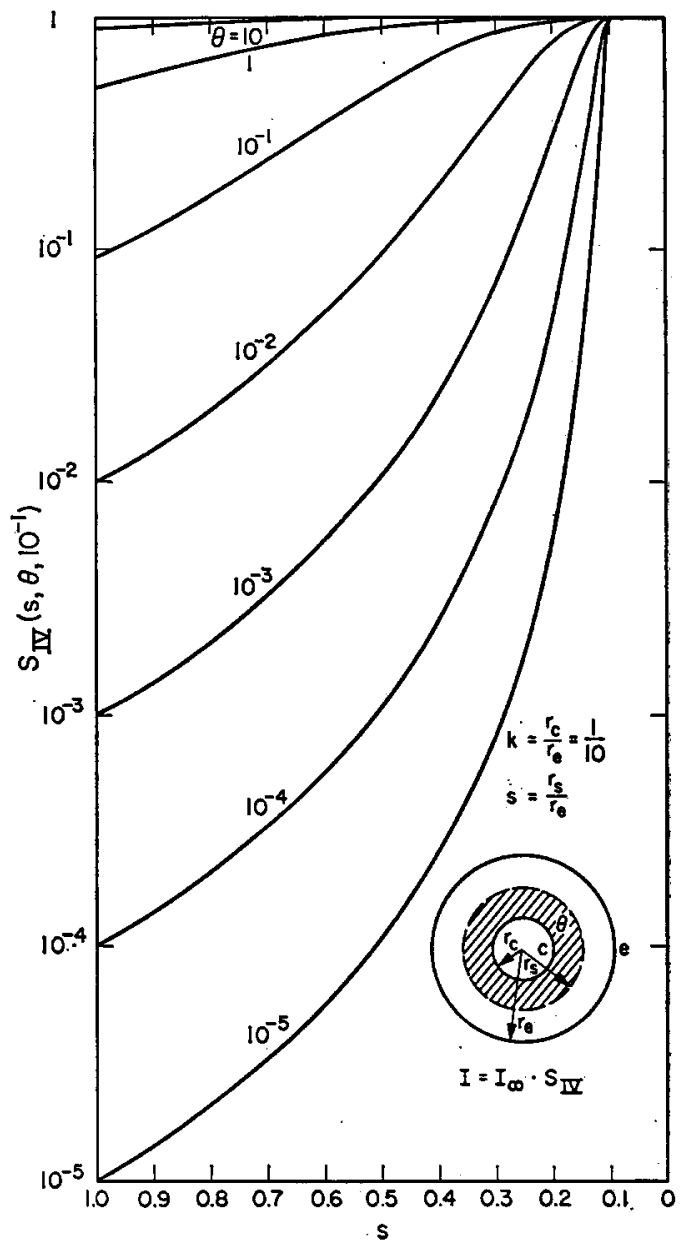

FIG. 15. Shallow traps of trapping parameter $\theta$ distributed uniformly over a limited distance in front of the collector reduce sclc by a factor $S_{\mathrm{IV}}(s, \theta, k)$ in structures of spherical geometry with the collector at the inner electrode.

The solution with the emitter outside is

$$
F=S_{\mathrm{IV}} \equiv \frac{\left[\int_{k}^{1}\left(1-z^{3}\right)^{\frac{1}{2}} z^{-2} d z\right]^{2}}{\left\{\int_{s}^{1}\left(1-z^{3}\right)^{\frac{1}{2}} z^{-2} d z+\int_{k}^{s}\left[1-s^{3}+\left(1+\theta^{-1}\right)\left(s^{3}-z^{3}\right)\right]^{\frac{1}{3}} z^{-2} d z\right\}^{2}} .
$$

Values of $S_{\mathrm{IV}}(s, \theta, k)$ are plotted in Fig. 15 for $k=10^{-1}$ and various $\theta$.

\section{APPROXIMATE TREATMENTS}

Figures 1 and 3 show that a uniform layer of traps reduces sclc much more efficiently when located in the immediate vicinity of the emitter rather than in front of the collector. The decrease of current caused by traps in front of the emitter is shown in detail in Fig. 2. Corresponding plots for cylindrical and spherical structures are given in Figs. 6 and 7 and Figs. 12 and 13 , respectively. In all these figures the abscissa repre- sents the thickness $t$ of the uniform layer of traps in normalized units. In all cases the trapping factor $F$ is observed to be proportional to $\theta / t$ as long as $\theta<1$ and $t$ does not exceed several tenths of the full base width.

The reason for the pronounced effect of traps in the vicinity of the emitter is of geometrical origin. Imagine a single trap in the base of a structure through which sclc flows. A charge captured by the trap does not contribute to the current, but adds to the voltage drop by virtue of its field $E_{t}$. The amount of this contribution is given by the line integral $\mathcal{S} E_{t} d r$ taken from the position of the trap to the collector boundary. This integral will be the larger the longer the path of integration, that 


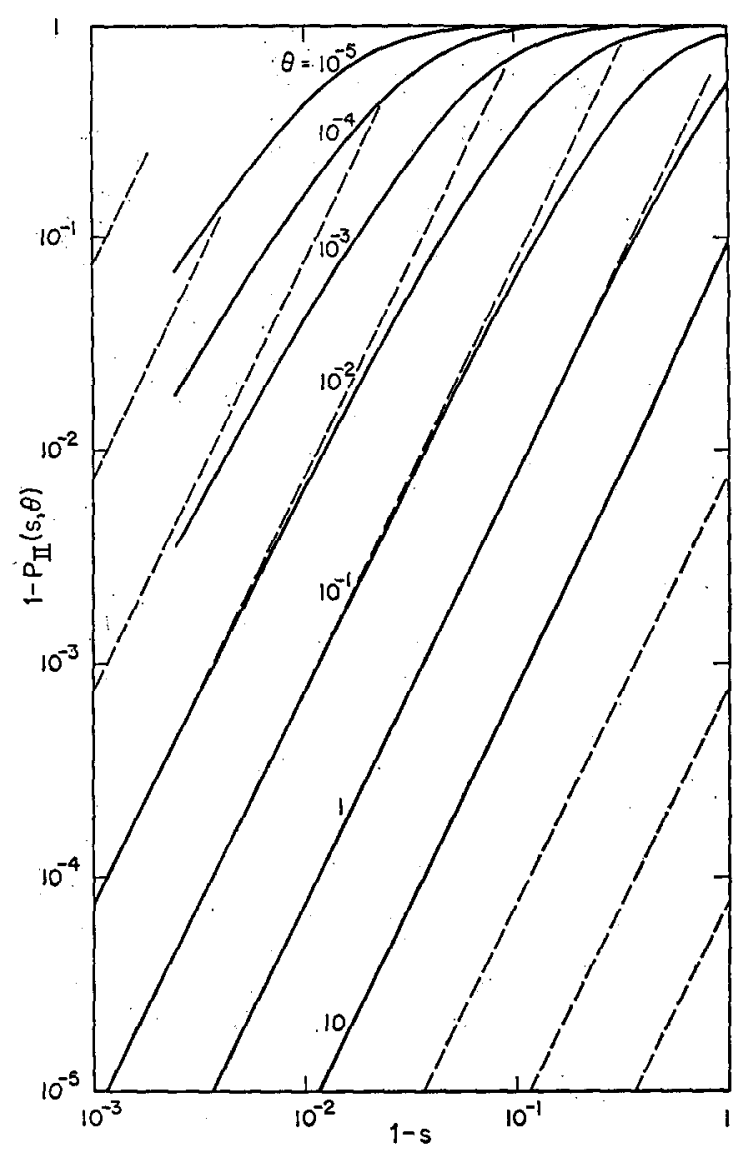

FIG. 16. The result of the approximate treatment (dashed lines) of $P_{\mathrm{II}}(s, \theta)$ compares well with the accurate solution (full lines) of Fig. 3 .

is, the closer the trap is located to the emitter boundary. In addition to this, shallow traps will capture more free charge carriers in the vicinity of the emitter than elsewhere because the concentration of free charge carriers is largest there. This effect is particularly pronounced for structures in which the area of the emitter is significantly smaller than that of the collector as, for example, in cylindrical and spherical structures with the emitter located at the center electrode. But the electric field set up by the trapped charge is then also weaker due to its spread over larger areas towards the collector. These two tendencies cancel and there results a common dependence for planar, cylindrical, and spherical structures.

These ideas are incorporated in the following simple model. The thin and uniform layer of traps in the vicinity of the emitter is taken as the main seat of space charge in the base. As long as the thickness $t$ of this layer is sufficiently small, curvatures in the emitter surface can be neglected and the $V-I$ characteristic of the layer is given by the relationship of sclc in a planar structure.

$$
I=\epsilon \epsilon_{0} \mu V_{t}^{2}\left(9 A / 8 t^{3}\right) \theta
$$

where $V_{t}$ is the voltage drop across this range and $A$ is the area of the emitter. The divergence of the electric field in the remaining trap-free part of the base is neglected. The field there is then essentially that of a capacitor of equivalent geometry; hence, for a planar structure

$$
E=\left(V-V_{t}\right) /(W-t) \text {. }
$$

The voltage drop, on the other hand, is developed almost entirely across the trap-free region because the field is initially zero at the emitter and $t$ is small; thus $E \approx V / W$. This field and that generated by the divergence in the layer are equal; hence

$$
\frac{3}{2}\left(V_{t} / t\right)=V / W \quad \text { or } \quad V_{t}=\frac{2}{3}(t / W) V
$$

which establishes the connection between $V$ and $V_{t}$ and confirms that $V_{l} \ll V$ as long as $k \ll W$. Substitution of $V$ into Eq. (8) yields

$$
I \approx \epsilon_{0} \mu V^{2} A \theta / 2 W^{2} t
$$

or

$$
I=\epsilon \epsilon_{0} \mu V^{2}\left(9 A / 8 W^{3}\right) P_{\mathrm{I}}(s, \theta), \quad P_{\mathrm{I}}(s, \theta) \approx(4 / 9) \theta / s .
$$

This estimated dependence of $P_{\mathrm{I}}$ is represented in Fig. 2 by dashed lines and seen to approximate the real dependence closely for small $\theta$ and $t$. Similar considerations show that the current is approximately given by

$I \approx \epsilon \epsilon_{0} \mu V^{2}\left(2 \pi l / r_{e}^{2}\right)\left[\theta / 2(s-1) \ln ^{2}(k)\right], \quad(1 \leq s \leq k)$

for a cylindrical structure with the emitter located at the inner electrode and by

$I \approx \epsilon \epsilon_{0} V^{2}\left(2 \pi l / r_{e}^{2}\right)\left[\theta / 2(1-s) \ln ^{2}\left(k^{-1}\right)\right], \quad(0<k \leq s \leq 1)$

in the opposite case. The corresponding results for a spherical structure are

$$
I \approx \epsilon_{0} \mu V^{2}\left(2 \pi / r_{e}\right)\left[\theta /(s-1)\left(1-k^{-1}\right)^{2}\right], \quad(1 \leq s \leq k)
$$

and

$I \approx \epsilon_{0} \mu V^{2}\left(2 \pi / r_{e}\right)\left[\theta /(1-s)\left(k^{-1}-1\right)^{2}\right], \quad(0<k \leq s \leq 1)$.

The dashed lines in Figs. 6, 7, 12, and 13 correspond to these approximate solutions.

If, on the other hand, the layer of traps is located in front of the collector, the trapped charge will change little on the $V-I$ characteristic of the structure but will, to a first approximation, merely generate an additional small voltage drop $V_{t}$, the value of which is approxmately

$$
V_{t}=\left[\left(\rho_{c} / \theta\right) / 2 \epsilon \epsilon_{0}\right] t^{2},
$$

where $\rho_{c}$ is the free charge carrier concentration at the collector in the absence of traps. The $V-I$ characteristic thus is

$$
I \approx \epsilon_{0} \mu\left(V-V_{t}\right)^{2} \delta=I_{\infty}\left(1-V_{t} / V\right)^{2},
$$

hence $\left(V_{l} \ll V\right)$

$$
F(\delta, \theta) \approx 1-2 V_{t} / V
$$


For a planar structure $\rho_{c}=\frac{3}{4}\left(\epsilon \epsilon_{0} V / W^{2}\right)$ so that $(t=1-s)$

$$
P_{\text {II }}(s, \theta) \approx 1-\left(\frac{3}{4}\right)(1-s)^{2} / \theta .
$$

This approximate result is compared with the accurate one in Fig. 16. The approximation is good as long as $(10 s) / \theta \leq 1$. This condition is equivalent to the requirement that the trapped charge in the layer should not exceed the free charge in the base. The corresponding approximate dependences for the cylindrical and spherical structures are

$$
\begin{array}{rr}
C_{\mathrm{II}}(s, \theta, k) \approx 1-(1+\pi / 2 k) \cdot(k-s)^{2} / k^{2} \theta & (1 \ll s \leq k), \\
C_{\mathrm{IV}}(s, \theta, k) \approx 1-\left(\ln \left(2 k^{-1}\right)-1\right)^{-1} \cdot(s-k)^{2} / \theta & (k \leq s \ll 1), \\
S_{\mathrm{II}}(s, \theta, k) \approx 1-\left(\frac{3}{2}\right)\left[\left(6 \pi / r_{e} \delta^{s, i}\right)\left(k^{-1}-k^{-4}\right)\right]^{-\frac{1}{2}} & \\
\cdot(k-s)^{2} / k^{2} \theta & (1<s \leq k), \\
S_{\mathrm{IV}}(s, \theta, k) \approx 1-\left(\frac{3}{2}\right)\left[\left(6 \pi / r_{e} \delta^{s, o}\right)\left(1-k^{3}\right)\right]^{-\frac{1}{2}} & \\
\cdot(s-k)^{2} / \theta & (k \leq s<1) .
\end{array}
$$

The quality of these approximations follows closely that of Fig. 16, as detailed comparisons show.

\section{DISCUSSION AND CONCLUSION}

The preceding considerations point out the dominating character of traps in the vicinity of the emitter. According to Figs. 1 or 3 , for example, the $V-I$ characteristic of a planar structure with a uniform distribution of traps extending throughout the base and with a trapping parameter $\theta=10^{-3}$ can be interpreted equally well by a uniform layer of traps of a parameter $\theta=10^{-4}$ extending only $4.6 \%$ into the base or by traps with a parameter $\theta=10^{-5}$ uniformly distributed in a sheath of a mere $0.46 \%$ penetration, while the remaining part of the base is free of traps. The approximate treatment given above shows that the magnitude of this effect is due to the fact that an emitter with a layer of shallow traps in its front actually operates in a mode of emission saturation. This conclusion is obviously independent of the specific spacial distribution of traps assumed in the argument. So is the conclusion that shallow traps outside of the immediate neighborhood of the emitter are of little significance, except possibly for large peaks in the trap distribution. Such peaks are most likely to occur in connection with large lattice imperfections, of which the crystal surfaces are the most prominent ones. The influence of surface states on sclc thus appears as a topic of particular interest, but has largely escaped attention so far. The approximate treatment also shows that structures of nonplanar geometry can be analyzed with planar models to a degree that should appear adequate for all practical purposes.

Even though these conclusions have been obtained for shallow traps only, it is clear that most statements will retain some degree of validity in more general cases. This means that the true nature of traps can only be determined when sufficient information is available on the spacial distribution of the traps.

Details on this distribution can be obtained from measurements of the $V-I$ characteristics of devices with different base widths. But it is often difficult to produce different samples with the necessary assurance that no other changes have been introduced also. This is the reason why such measurements have been performed only rarely. If several species of traps with different spacial distributions are present simultaneously, the interpretation of such measurements is not simple either. The transient response of a device also contains information on the spacial distribution of traps. Unfortunately, the interpretation is difficult and probably not unique in most cases, because relaxation times are introduced simultaneously as an additional parameter. External influences such as strain, radiation, and temperature can also be used as tools of investigation, but their application is often limited or involved, or both. To characterize a trap unambiguously is not a simple task.

\section{ACKNOWLEDGMENTS}

M. H. Kryder and G. H. Parker have been helpful in collecting data for Tables I and II. The U. S. Naval Ordnance Test Station, Pasadena Annex, and the Jet Propulsion Laboratory supported the work in parts. The author acknowledges and thanks for this personal help and financial support. 